\section{La Medicina Familiar y Comunitaria como fuente de cuidados de Salud Mental}

\section{Family and Community Medicine as source of Mental Health Care Medicina Familiar e Comunitária como fonte de cuidados em Saúde Mental}

\section{Grupo de Trabajo:}

Amanda Astudillo (Colombia), Alfonso Avila (Colombia), Garibaldi Baldovino (Colombia); Virginia Cardozo (Uruguay), Ximena Cruz (Bolivia), Carmen Daza (Colombia), Sonia De La Portilla (Colombia), José Ignacio Díaz (Colombia), Ysabel Díaz (Rep. Dominicana); Giuliano Dimarzio (Brasil), Elizabeth Escobar (Colombia), Margarita García (Colombia), Luz Girón (Colombia), Carlos Guevara (Colombia), Melissa Gutiérrez (Colombia), Jakeline Jolkh (Colombia), Fátima González (Paraguay), Carlos Guevara (Colombia), Giuliano Dimarzio (Brasil), Mauricio Molina (Colombia), Marta A. Mejía (Venezuela), Alvaro Pérez (Colombia), Marcela Pérez (Chile), Olga Polo (Perú), Lina Quintero (Colombia), Katherine Rocha (Colombia), Diana Rodríguez (Colombia), Julieth Salazar (Colombia), Martha Sánchez (Bolivia), Mónica Sánchez (Colombia), Gladys Sandoval (Paraguay), Heyder Satizabal (Colombia), Melba Vásquez (Costa Rica), José Manuel Vivas (Colombia), Vilma Velásquez (Colombia).

\section{Resumen}

En la Séptima Cumbre Iberoamericana de Medicina Familiar, Cali - Colombia 2018, el grupo de trabajo Salud Mental (SM) reflexionó sobre como la Medicina Familiar (MF) puede actuar para apoyar a las personas que enfrentan situaciones de estrés frente a la vida diaria, conflictos (armados/no armados), emergencias y desastres naturales. Estudio descriptivo de corte transversal basado en una encuesta de 42 preguntas a 99 profesionales sanitarios iberoamericanos provenientes de 15 países, 98 médicos y 1 psicólogo, $8 \%$ residentes de MF, 85\% especialistas en MF, $4 \%$ médicos generales, $2 \%$ psiquiatras y $1 \%$ internista. El $47 \%$ de los médicos percibe como buena la capacidad de los médicos de familia en el abordaje de la SM. En cuanto a los problemas de SM observados, $30 \%$ indica Trastorno de Ansiedad, $27 \%$ depresión, 17\% insomnio, 10\% alcoholismo, $7 \%$ adicción a drogas ilícitas, $5 \%$ trastornos alimentarios y $4 \%$ trastorno de estrés postraumático. En este contexto se realizaron las recomendaciones para la Carta de Cali que consideran la formación en SM necesaria para los Médicos Familiares, con estrategias de autocuidado costo efectivas, mediante el fortalecimiento del trabajo comunitario. El cuerpo docente de las residencias de MF debe hacerse cargo de acciones tendientes al autocuidado de los alumnos, propendiendo a facilitar el proceso de aprendizaje y la preparación para ejercer la profesión en un medio tan complejo como son los centros del primer nivel de atención o en cualquier contexto en el que se trabaje con la estrategia de Atención Primaria de Salud.

Palabras clave: Medicina Familiar y Comunitaria; Salud Mental; Emergencias; Desastres
Macarena Moral Lópeza

Diana Yuruhán Mohrbach ${ }^{b}$

Carmen Ruiz Puyana ${ }^{c}$

Maria Inez Padula Anderson ${ }^{\mathrm{d}}$

Paula Andrea Carmona Mejía

Sandra Fortes ${ }^{\dagger}$

José Rubén Quiroz ${ }^{9}$

María Luisa Vera González ${ }^{h}$

a Facultad de Medicina, Universidad de Chile; Confederación Iberoamericana de Medicina Familiar (CIMF/WONCA); Sociedad Chilena de Medicina Familiar (SOCHIMEF). Chile. moralmacarena@hotmail.com (Autora correspondiente)

b Universidad Católica "Nuestra Señora de la Asunción" (UC); Universidad del Norte (UN); Sociedad Paraguaya de Medicina Familiar (SPMF). Paraguay. dyuruhan@ @otmail.es

c Universidad Militar Nueva Granada (UMNG) Universidad El Bosque (UEB); Hospital Militar Central; Sociedad Colombiana Medicina Familiar (SOCMEF). Colombia. cruizpuyana@gmail.com

d Faculdade de Ciencias Médicas, Universidade do Estado do Rio de Janeiro (UERJ); Confederación Iberoamericana de Medicina Familiar (CIMF). Rio de Janeiro, RJ, Brasil. inezpadula@gmail.com

e Universidad de La Coruña (UDC); Sociedad Chilena de Medicina Familiar (SOCHIMEF). España. pa.carmona@ hotmail.com

' Faculdade de Ciencias Médicas, Universidade do Estado do Rio de Janeiro (UERJ); Guía de Intervención del Mental Health Gap, Organización Panamericana de Salud (OPS/OMS). Brasil. sandrafortes@gmail.com

${ }^{g}$ Instituto de la Familia Asociación Civil (IFAC). México. joserubenquiroz@gmail.com

${ }^{\mathrm{h}}$ Caja Nacional de Salud (CNS). Bolivia maluverag@gmail.com

Financiación: ninguna declarada. Aprobación ética: no aplicable.

Conflicto de intereses: ninguna declarada. Procedencia y revisión por pares: revisado por pares. Recibido el: 25/07/2018. Aceptado el: 27/08/2018. 


\begin{abstract}
During the Seventh Iberoamerican Summit of Family Medicine, Cali Colombia 2018, the Mental Health (MH) working group reflected on how Family Medicine (FM) can act to support people facing stressful situations in daily life as well as in conflicts (armed/unarmed), emergencies and natural disasters. Descriptive cross-sectional study, based on a survey of 42 questions to 99 lberoamerican health professionals from 15 countries; 98 physicians and 1 psychologist. $8 \%$ residents of family medicine, $85 \%$ family physicians (FP), $4 \%$ general doctors, $2 \%$ psychiatrists and $1 \%$ internists $47 \%$ of physicians perceive as good the ability of FP in the approach to $\mathrm{MH}$. Concerning the $\mathrm{MH}$ problems observed, $30 \%$ where anxiety disorder, $27 \%$ depression, $17 \%$ insomnia, $10 \%$ alcoholism, $7 \%$ illicit drug abuse, $5 \%$ eating disorders and $4 \%$ post-traumatic stress disorder. Wich such results, recommendations for the Cali Declaration consider the necessary $\mathrm{MH}$ training for Family physicians, with cost-effective self-care strategies through strengthening community work. The teachers of FP must take actions tending to the self-care of their students, to facilitate the learning process and the preparation to practice the profession in such a complex environment as the Primary Care (PC) centers or in any place based on PC strategy.
\end{abstract}

Keywords: Family Practice; Mental Health; Emergencies; Disasters

\title{
Resumo
}

Na Sétima Cúpula Ibero-Americana de Medicina Familiar, Cali - Colombia 2018, o grupo de trabalho Saúde Mental (SM) refletiu sobre como a Medicina de Família (MF) pode atuar em para apoiar a saúde integral das pessoas que enfrentam situações de estresse na vida diaria, como conflitos armados/desarmados, emergências e desastres naturais. Estudo descritivo transversal com base em um levantamento de 42 perguntas a 99 profissionais de saúde ibero-americanos provenientes de 15 países; 98 médicos e 1 psicólogo. $8 \%$ de residentes de MF, $85 \%$ de especialistas em MF, $4 \%$ de clínicos gerais, $2 \%$ de psiquiatras e $1 \%$ de internistas. $47 \%$ dos médicos percebem como boa a capacidade dos médicos de família na abordagem da SM. Em relação aos problemas SM, 30\% indicam Transtorno de Ansiedade, $27 \%$ de depressão, $17 \%$ de insônia, 10\% de alcoolismo, $7 \%$ de dependência de drogas ilícitas, $5 \%$ de transtornos alimentares e $4 \%$ de transtorno de estresse pós-traumático. Neste contexto, foram feitas recomendações para a Carta de Cali que consideram o treinamento de SM necessário para médicos de família, com estratégias de autocuidado custo-efetivas através do fortalecimento do trabalho comunitário. O corpo docente das residências do MF deve se encarregar de ações que promovam o autocuidado dos alunos, visando facilitar o processo de aprendizagem e o preparo para a prática da profissão em um ambiente tão complexo como os centros de Atenção Primária à Saúde (APS) ou em qualquer contexto em que se trabalhe com a estratégia APS.

Palavras-chave: Medicina de Família e Comunidade; Saúde Mental; Emergências; Desastres

\section{Introducción}

La Séptima Cumbre Iberoamericana de Medicina Familiar, Cali Colombia 2018, a través del grupo que tuvo como lema "La Medicina Familiar y Comunitaria como fuente de cuidados de Salud Mental", nos dio la oportunidad de reflexionar sobre el cómo la medicina familiar puede y debe actuar en relación a la problemática de Salud Mental (SM), buscando aportes concretos conforme al lema central de la cumbre: "Medicina Familiar y Políticas Públicas en territorios de equidad y paz".

En el resumen ejecutivo sobre Prevención del Suicidio de la Organización Mundial de la Salud (OMS) de 2014, se plantea que más de 800.000 personas se suicidan al año y que por cada persona que se suicida habría al menos otras 20 personas que han intentado suicidarse. Es además la segunda causa de muerte entre las personas de 15 a 29 años. ${ }^{1}$ Habría entonces cada año 16 millones de personas en riesgo vital por intento suicida por patologías de Salud Mental en el mundo. Por otro lado, el principal factor de riesgo frente al suicidio es un intento suicida previo, por lo que esas 16 millones de personas tienen potencialmente un riesgo de muerte mayor que el común de la población.

Las causas son múltiples y relacionadas con crisis que determinan una disminución de la capacidad para afrontar las tensiones de la vida tanto aquellas de la cotidianeidad (problemas financieros, rupturas de relaciones, dolores y enfermedades crónicas, etc.), como las de situaciones más complejas: las emergencias y los desastres ${ }^{1}$ tanto naturales (inundaciones, terremotos, tsunamis, etc.) como antrópicos ${ }^{2}$ (conflictos políticos y/o armados, incendios, etc.). 
Incluso se ha acuñado el término tsunami de la salud mentaß ${ }^{\beta}$, en el contexto del desarrollo de los niños y adolescentes que son víctimas de violencia física o sexual grave, que inician carreras delictuales alrededor de los 13 años dependiendo de los países y que aumentan progresivamente el consumo de drogas y alcohol. Una amenaza latente para el futuro de nuestras naciones.

Por la forma de vida y las situaciones creadas o no por el ser humano, se está produciendo un contexto en el que un número importante de personas es potencialmente incapaz de afrontar las dificultades de la vida diaria y determina una mayor incapacidad para enfrentar las dificultades inherentes a emergencias y desastres, en particular las antrópicas, que son aquellas en las que el ser humano es la causa directa; y en especial, las determinadas por conflictos políticos, armados o no armados.

Si bien toda la información anterior pudiera parecer apocalíptica, el informe de la OMS de 2013: "Volver a construir mejor"4 plantea como paradoja que las emergencias son una oportunidad para volver a construir mejor el sistema de atención en SM, sin importar lo débil que haya sido el sistema antes de la emergencia ni la gravedad de la misma. Se comenta que las emergencias y los desastres, naturales o antrópicos pueden dar lugar a situaciones donde la SM requiere especial consideración debido a tres problemas comunes:

- aumento de las tasas de problemas de SM,

- la debilidad de la infraestructura de SM y

- las dificultades que se generan en la coordinación de los organismos que proveen servicios en el área de la SM.

En estas situaciones, la prevalencia de la depresión y de los trastornos por estrés postraumático aumenta sustancialmente. Se produce también un aumento de la vulnerabilidad y las necesidades de las personas que ya tenían trastornos mentales graves como esquizofrenia, trastorno bipolar, ansiedad y dependencia a alcohol y drogas.

Como resultado de las emergencias y desastres puede debilitarse la infraestructura de atención en SM. Hay mayor necesidad de servicios y los mismos trabajadores de la salud pueden ser víctimas de la emergencia, en muchos casos necesitan cuidar de sus propias familias o amigos antes de cumplir con sus deberes profesionales. Esto puede llevar consigo una escasez de trabajadores de salud calificados.

En emergencias mayores con inicio agudo pueden crearse situaciones caóticas por la aparición de múltiples agencias de apoyo. Frente a este gran impulso inicial de organizaciones de todo tipo, gubernamentales y no gubernamentales debe ponerse especial hincapié en la coordinación de la oferta de servicios. El rol fundamental de la ayuda humanitaria es fortalecer las estructuras públicas, logrando así coordinar las acciones y la sostenibilidad a largo plazo de la estructura funcional de los servicios de salud. ${ }^{5}$ La importancia de la sostenibilidad a largo plazo radica en que no sólo se logra un mejor estado de salud general, sino que también mayor educación, mayor productividad y mejores relaciones interpersonales, y por ende una mejor calidad de vida. Con este actuar no sólo se beneficia la SM de las personas, sino que también el funcionamiento general del país afectado y la resiliencia de la sociedad que es capaz de recuperarse de una situación de emergencia. Las acciones en el marco de la recuperación temprana sientan las bases para una mentalidad a largo plazo.

La atención en SM debe centrarse en los servicios que son accesibles a la comunidad. En el mencionado informe de la OMS se plantea que la descentralización de los sistemas de SM hacia sistemas 
de cuidados basados en la comunidad es una estrategia fundamental. En especial para hacer frente a situaciones de emergencias y desastres. Es necesario para la recuperación temprana, que las directrices de los organismos a cargo generen estándares basados en el consenso entre los servicios de salud y la comunidad. La comunidad debe constituirse en actor estratégico. Es importante destacar que la mayor inversión debe realizarse en las personas, comunidad y trabajadores de la salud, más que en infraestructura.

Se debe fortalecer y expandir la atención en SM basada en la comunidad, debe redefinirse el actuar de los centros hospitalarios psiquiátricos de larga estadía, considerando incluso disminuir su tamaño. Ningún nivel de servicio, de primario a secundario y terciario, puede cumplir todas las necesidades de salud mental, es necesario buscar la combinación adecuada entre todos ellos. El autocuidado, el cuidado informal de la comunidad y la atención primaria en SM son la base del modelo piramidal de cuidados. Es a este nivel donde debieran coordinarse la mayor parte de ellos. Para aquellos que requieren servicios más intensivos en algún momento de su vida, el nivel secundario debiera proveer de atención ambulatoria e internación de corta estadía. Entendiendo al nivel secundario como centros comunitarios de salud mental y hospitales generales. Sólo una fracción muy pequeña de las personas con problemas severos de SM necesita hospitalizaciones de larga estadía, considerando un nivel terciario, pero sin que por ello se considere estructurar hospitales psiquiátricos de estadía permanente. La meta es siempre lograr atención y control ambulatorio. En todos los niveles, el modelo enfatiza que las personas con problemas de SM deben participar en la autogestión de sus condiciones. ${ }^{5}$

En este contexto general un informe del 2017 de la Organización para la Cooperación y el Desarrollo Económico (OECD) sobre la SM $\mathrm{SM}^{5}$ plantea que se debe promover e invertir en la introducción de programas que promuevan la buena salud mental y la prevención de enfermedad mental. El establecer acciones que prevengan la depresión y la ansiedad conlleva beneficios económicos para las familias, mientras que ciertas intervenciones en el lugar de trabajo pueden reducir en más de un tercio el costo de la baja productividad. Se observa, sin embargo, un compromiso gubernamental desigual entre la promoción de la salud mental y otras patologías en los sistemas de salud, con tendencia a inclinarse hacia las últimas, como son por ejemplo el fuerte foco en la promoción de patologías cardiovasculares.

El documento de la OECD realiza varias recomendaciones para los gobiernos de los países, entre ellas:

- Implementar programas integrados de salud mental, herramientas de aplicación y política laboral;

- Priorizar el bienestar mental de los ciudadanos de todas las edades. Para algunos países esto puede demandar actividades a lo largo de la vida del individuo y para otros implicará promover esfuerzos enfocados a grupos particulares como ancianos o desempleados. Se puede realizar mediante un enfoque escalonado de la acción haciendo uso de nuevas oportunidades, como son por ejemplo los sistemas informáticos en línea.

- Desarrollar y respaldar una estrategia permanentemente actualizada y exhaustiva para promocionar la SM a lo largo de la vida. Basada en la mejor evidencia de la efectividad de las intervenciones con foco en el contexto local.

- Monitorizar y evaluar en forma permanente la efectividad de lo implementado, de modo tal que permita realizar cálculos estimativos del retorno económico de lo invertido en actividades de promoción y prevención. Esto puede mejorar la comprensión del costo/efectividad de las 
inversiones orientadas a mejorar la SM de las personas, favoreciendo que otros actores, externos al sector de la provisión de atención de salud, visualicen mejor el retorno económico de ésta inversión, impulsándolos a invertir y participar en la realización de actividades de promoción y prevención.

- Establecer redes de colaboración intersectorial, reconociendo su importancia más allá de los sistemas de cuidado de la salud y de la SM, involucrando en la promoción y prevención en SM a otros sectores interesados incluyendo asistencia social, sistemas educativos y lugares de trabajo.

En el informe de la Organización Panamericana de la Salud (OPS 2013) sobre los sistemas de SM en América Latina y El Caribe ${ }^{6}$ se refleja la importancia de abarcar los trastornos mentales y neurológicos desde el ámbito de la Atención Primaria en Salud (APS), ya que representan casi la cuarta parte de la carga total de enfermedad en América Latina y el Caribe. "Un sistema de salud mental eficiente es vital tanto para poder ofrecer una respuesta apropiada y reducir esa carga que se traduce en morbilidad, mortalidad y discapacidad, como para cerrar la alta brecha de personas enfermas que no están recibiendo ningún tipo de tratamiento". ${ }^{7}$

Se desarrolla la evaluación de los sistemas de atención en SM desde la relevancia de su reestructuración. En el informe se reconoce que en América del Sur, América Central, México y el Caribe latino, la formación en pregrado de medicina y enfermería, además de las capacitaciones en el ambiente laboral en APS, relacionadas con la dedicación y carga horaria con respecto a la salud mental, son bajas en general, siendo la capacitación en servicio levemente mejor en América del Sur.

La formación y capacitación en servicios de APS son al menos insatisfactorias, no permiten dar respuesta adecuada a la problemática de SM. Son insuficientes para mejorar la capacidad de resolver frente a la demanda de atención por problemas psicosociales y de SM. La integración de la SM en los servicios de APS por lo general es limitada. Esto restringe considerablemente la capacidad de la APS para cumplir con las funciones en relación a la SM y el nivel de resolutividad que se le confía en el contexto de un modelo comunitario de salud mental.

Según éste informe la disponibilidad de protocolos de evaluación y tratamiento es muy dispar, de casi inexistente a no necesariamente disponibles o conocidos según la región. Se suma además la escasa interacción y limitada integración, a veces por falta de información, de los profesionales de APS con profesionales especializados en SM y con agentes del sistema alternativo de atención (medicina complementaria integrativa).

Respecto del plan terapéutico a instaurar a las personas que consultan en APS por problemáticas de SM, como plantea el documento, es evidente que el acceso a los psicofármacos es una condición necesaria para que pueda cumplir la función de atenderlas apropiadamente. En ese contexto se informa que los medicamentos sí están disponibles, aunque, en muchos casos, de manera parcial.

En cuanto a los recursos humanos los datos muestran la escasez de los mismos y la desigual distribución en los países, con una marcada variabilidad entre las subregiones.

Es importante hacer mención de los factores que limitan el acceso a la salud mental son entre otros:

- la distribución entre el sistema privado y público de los profesionales capacitados en SM, muchas veces en desmedro del sistema público, donde se concentra a la mayor parte de la población, 
- el desequilibrio en la distribución del personal capacitado en SM con tendencia hacia mayor cantidad en los hospitales psiquiátricos, con excepción de América del Sur en la que se está iniciando una tendencia a la existencia de mayor cantidad de personal capacitado en centros de atención ambulatoria intermedia, pero sin que exista aún una tendencia comparable en la APS,

- una distribución desigual de los psicofármacos disponibles, y

- una distribución desigual geográfica del personal capacitado, en que la mayoría se concentra en las ciudades y en especial en las capitales de los países de la región.

Otro aspecto fundamental en el desarrollo de estrategias para la intervención en patologías de SM es el rol de la sociedad civil - comunidad, asociaciones de usuarios y familiares. Si bien el documento de la OMS sobre la región plantea que por el momento es muy limitado e incluso inexistente (no participa ni en la discusión ni en la toma de decisiones respecto de la prestación de servicios de salud mental), otros documentos revisados en éste análisis destacan la importancia de la participación de las comunidades, en especial en situaciones de emergencia y desastres. Quizás entonces falta desarrollar dispositivos que permitan la participación de los usuarios en contextos habituales, ya que en situaciones de emergencias y desastres, la comunidad adquiere un rol espontáneo y vital.

Frente al rol espontáneo y vital de la sociedad civil es que surge la necesidad de generar estrategias fáciles de implementar para el manejo de problemas y enfermedades de SM, tanto en el diario vivir como en situaciones de emergencias y desastres. Es así como se desarrollan estrategias como el "Programa de Acción para superar las Brechas en SM: mhGAP" (por sus siglas en inglés: mental health Gap Action Program). Este programa fue lanzado en el 2008 por la OMS como una forma de enfrentar el gran desafío de desarrollar estrategias de bajo costo, accesibles a la población, en especial de países de ingresos bajos a medios y en particular en situaciones de emergencias humanitarias. Es así que surgen diferentes guías y programas de fácil implementación y accesibles a personas sin entrenamiento formal o con recomendaciones muy específicas del entrenamiento requerido.

Existen por tanto guías clínicas para profesionales de la salud que permiten un apoyo rápido y eficaz en situaciones humanitarias. Una de ellas, es la Guía de Intervención Humanitaria mhGAP (GIH - mhGAP). ${ }^{8}$ Se trata de una guía clínica básica sobre los trastornos mentales, neurológicos y por uso de sustancias dirigida a los trabajadores del área de la salud: médicos generales, enfermeras, parteras y auxiliares clínicos, así como médicos con especialidades ajenas a la psiquiatría o la neurología que trabajan en servicios no especializados, en particular para los países de ingresos bajos y medianos. Contiene consejos para los directores de servicios clínicos respecto de principios generales de atención aplicables a las emergencias humanitarias, relevando la importancia del apoyo multisectorial.

Plantea los siguientes principios generales de la atención a personas con trastornos mentales, neurológicos y por uso de sustancias en emergencias humanitarias:

1. Principio de la comunicación: Se pondera la comunicación directa, concisa, respetuosa confidencial, con escucha activa, incluyente para el paciente, y, si es necesario, con intérpretes capacitados.

2. Principio de evaluación: Se da importancia a la plena identificación del trastorno mental, neurológico o uso de sustancias y a la interpretación que le da el paciente a su problema de salud. Debe haber un interrogatorio que incluya antecedentes familiares, antecedentes de la persona, 
estrategias utilizadas para la resolución del problema y el apoyo social con el que cuenta. Se aconseja hacer preguntas sobre el suicidio de manera sensible.

3. Principio para el manejo: La capacitación y el entendimiento del manejo que tendrá el paciente por parte de los cuidadores.

4. Principio de la reducción del estrés y del fortalecimiento del apoyo social: Fundamental es la reducción del estrés que puedan presentar el paciente o sus cuidadores. Se recomienda el uso de la Guía del IASC sobre salud mental y apoyo psicosocial en emergencias humanitarias y catástrofes, además de ejercicios de relajamiento con técnicas de respiración.

5. Principio de protección de los derechos humanos: Proteger los derechos de las personas con afecciones mentales o neurológicas e integrarlos a la comunidad.

6. Principio de la atención al bienestar general: Ayudar a las personas afectadas al acceso sin peligro a los servicios que necesiten para sobrevivir, llevar una vida digna y velar por la salud física general.

Orienta sobres los síntomas y signos del estrés agudo y postraumático, el duelo, la depresión mayor, la psicosis, la epilepsia, la adicción al alcohol y otros trastornos emocionales; mencionando los puntos importantes para la evaluación del cuadro clínico; y especifica un plan de manejo básico que incluye las intervenciones farmacológicas y las psicosociales.

Existen además programas de consejería breve orientada a problemas, de fácil implementación por profesionales de la salud no especializados en SM. Es el caso del Programa de Manejo de Problemas "Plus" - Ayuda, para adultos afectados por la angustia en comunidades expuestas a la adversidad, dentro del programa mhGAP ya descrito; programa conocido con las siglas $\mathrm{PM}+{ }^{9}$

La estrategia PM+ requiere que los profesionales tengan habilidades de ayuda básica, con foco en la comunicación y en el construir una relación con las personas que van a atender. Los Médicos de Familia, especialmente aquellos formados en el abordaje centrado en el paciente y en las habilidades comunicacionales, están especialmente preparados para ello. Por otra parte, el PM +, ayuda a mejorar las habilidades para tratar pacientes que han tenido que enfrentar situaciones vitales complejas.

Las intervenciones o estrategias conductuales consideradas son:

1. MANEJO DEL ESTRÉS: se utiliza la estrategia de respiración lenta, la más apropiada en la mayoría de las situaciones que producen ansiedad y estrés. Se puede combinar con métodos de relajación localizada cuando la situación es percibida como más compleja. Esta intervención conductual es introducida desde el inicio en el PM+ y debiera ser practicada al término de cada sesión.

2. MANEJO DE LOS PROBLEMAS: en situaciones en las que las personas se enfrentan a problemas prácticos (cesantía, conflictos familiares, etc.). El profesional y el usuario trabajarán en conjunto para considerar soluciones posibles al problema que más preocupa a la persona. Pueden plantear en conjunto soluciones para la resolución del problema y generar una estrategia para llevar a cabo las soluciones.

3. PONERSE EN MARCHA, MANTENERSE ACTIVO: el objetivo es recuperar y mantener el nivel de actividad, lo que tiene un impacto inmediato en el humor, ya que las personas con depresión con frecuencia dejan de hacer sus actividades usuales. 
4. FORTALECIMIENTO DEL APOYO SOCIAL: Ios individuos con problemas emocionales pueden aislarse de sus redes de apoyo tanto las personales como las organizacionales. Si las personas tienen una buena red de apoyo social y la usan con regularidad, puede que todo lo que se necesite es alentarlas a continuar haciéndolo. En el caso de las personas que no las tienen, puede que tome más tiempo analizar el cómo pueden mejorar sus redes de apoyo social y se les debe ayudar a que desarrollen un plan para que reciban más apoyo social.

5. MANTENERSE BIEN Y MIRAR AL FUTURO: de la misma manera que lo hacen las personas que se recuperan de heridas o enfermedades físicas, las personas con problemas o enfermedades mentales sufren "alzas" y "bajas" de sus emociones durante la recuperación. Es importante al finalizar las sesiones aclarar que el practicar las estrategias después de terminada la consejería es fundamental para mantenerse bien. En la eventualidad de que reaparezca una situación problemática que cause angustia, es probable que la persona sea capaz de responder utilizando estas estrategias.

\section{Objetivo General}

Analizar el rol del médico familiar en la Salud Mental en Iberoamérica con el fin de apoyar la salud integral de las personas que padecen trastornos a consecuencia de enfrentar situaciones de conflictos (armados o no armados), de emergencias y desastres naturales, así como los presentes en la cotidianeidad de la vida.

\section{Objetivos específicos}

1. Identificar el rol del médico familiar en la detección y el abordaje de los trastornos prevalentes de Salud Mental, así como la capacidad de apoyar la detección de trastornos en la población afectada por conflictos armados y no armados y situaciones de emergencia y desastres naturales, en Iberoamérica.

2. Conocer el rol del Médico Familiar en la detección precoz de trastornos de salud mental en todas las situaciones de la vida y en especial el estrés post traumático en población del post-conflicto y emergencias y desastres.

3. Analizar la disponibilidad de Médicos Familiares y de recursos humanos en general en los países de Iberoamérica para el abordaje de la problemática de salud mental en la población a cargo.

4. Reconocer la capacidad de participación de Médicos de Familia en el desarrollo de estrategias para el abordaje de problemáticas que se generan en conflictos armados y no armados, emergencias y desastres naturales.

5. Identificar las fortalezas del modelo de atención integral, centrada en la persona, su familia y la comunidad, como una forma permanente de detección precoz y manejo de problemáticas de salud mental en la práctica clínica habitual de los Médicos de Familia.

6. Identificar las destrezas con las que cuenta el Médico de Familia para la prevención de problemas de salud mental, de acuerdo al ciclo vital individual y familiar. 


\section{Método}

Estudio descriptivo de corte transversal, que recoge la opinión de diferentes profesionales de la salud en Iberoamérica sobre las capacidades propias y ajenas para abordar problemas de salud mental.

Para la recolección de la información, se diseñó una encuesta orientada por los objetivos del trabajo, que constó de 42 preguntas, 12 de identificación general (país, ciudad de residencia, edad, género, profesión, etc.), 21 preguntas cerradas y 9 abiertas, dirigidas a conocer la opinión, actitudes o experiencias, en relación a salud mental.

Para la validación del instrumento se realizó una prueba piloto que permitió la realización de correcciones por los autores principales del trabajo.

El link de la encuesta fue enviado vía correo electrónico a miembros de la Confederación Iberoamericana de Medicina Familiar, y se les solicitó que la promocionaran entre sus contactos.

Se estableció como criterio de inclusión que todos los encuestados fueran profesionales de la salud graduados o en formación de postgrado, excluyéndose los estudiantes en formación de pregrado.

Se obtuvieron 100 respuestas a la encuesta, de las cuales 99 cumplieron los criterios de inclusión y 1 no los cumplió por tratarse de 1 estudiante de pregrado en medicina. Las 99 restantes fueron respondidas por Médicos con o sin especialidad y 1 Psicólogo.

Al proceder al análisis de las respuestas de las 99 encuestas en 8 de ellas se suprimieron 2 preguntas al detectarse errores en la selección de las alternativas planteadas o en la interpretación de las preguntas abiertas. En esas preguntas el análisis se basó en 91 encuestas. El análisis final se basó en 99 encuestas, excepto en los ítems 16 y 17 donde se obtuvieron 91 respuestas.

\section{Revisión Bibliográfica}

Se realizó una revisión bibliográfica del tema de Salud Mental (SM) en situaciones de emergencias y desastres, naturales y antrópicas, y en contextos de la vida cotidiana, con búsqueda activa orientada a artículos que entreguen información global de SM (Regiones, Subregiones) y oriente hacia el manejo de problemas de SM.

El criterio de selección de la bibliografía fue fundamentalmente por la búsqueda de los últimos 10 años de informes de organizaciones de la salud y aportes de los mismos autores en relación a sus países de origen.

La revisión bibliográfica se realizó mediante la distribución de diferentes textos en grupos de interés. Se distribuyeron18 documentos entre los 12 interesados en participar, solicitando que realizaran un resumen de los documentos que les habían sido asignados. Se obtuvieron 9 resúmenes de los documentos distribuidos de 7 participantes.

\section{Presentación en Plenario de la Cumbre}

Se presentó en plenario en la Cumbre la temática de Salud Mental en Iberoamérica y las necesidades de abordaje, como una forma de introducir el tema para los el trabajo de grupo. 


\section{Trabajo de grupo}

Durante el evento de la Cumbre se procedió al trabajo de grupo para establecer recomendaciones sobre la problemática de Salud Mental para la Carta de Cali. Inicialmente se hizo una presentación sobre la temática de Salud Mental. Luego los participantes se distribuyeron en grupos pequeños ( 5 a 6 personas como máximo) con preguntas claves respecto de los objetivos del presente trabajo. Específicamente se les indicó proponer tres recomendaciones para la Carta de Cali. Al finalizar se realizó un catastro de todas las recomendaciones, agrupándolas según calidad y acción en el área de la Salud Mental, para finalizar con tres recomendaciones construidas en base a las recomendaciones.

\section{Resultados de la Encuesta}

De las 99 encuestas admitidas en el estudio, se desprende que los encuestados provenían de 15 (75\%) países que conforman la Confederación Iberoamericana de Medicina Familiar (CIMF), registrándose una abstención en el llenado del instrumento en 5 (25\%) países de la región (Cuba, El Salvador, Nicaragua, Portugal y República Dominicana). La participación por país fue desigual (Gráfica 1), determinando un sesgo que se deberá considerar en futuros estudios.

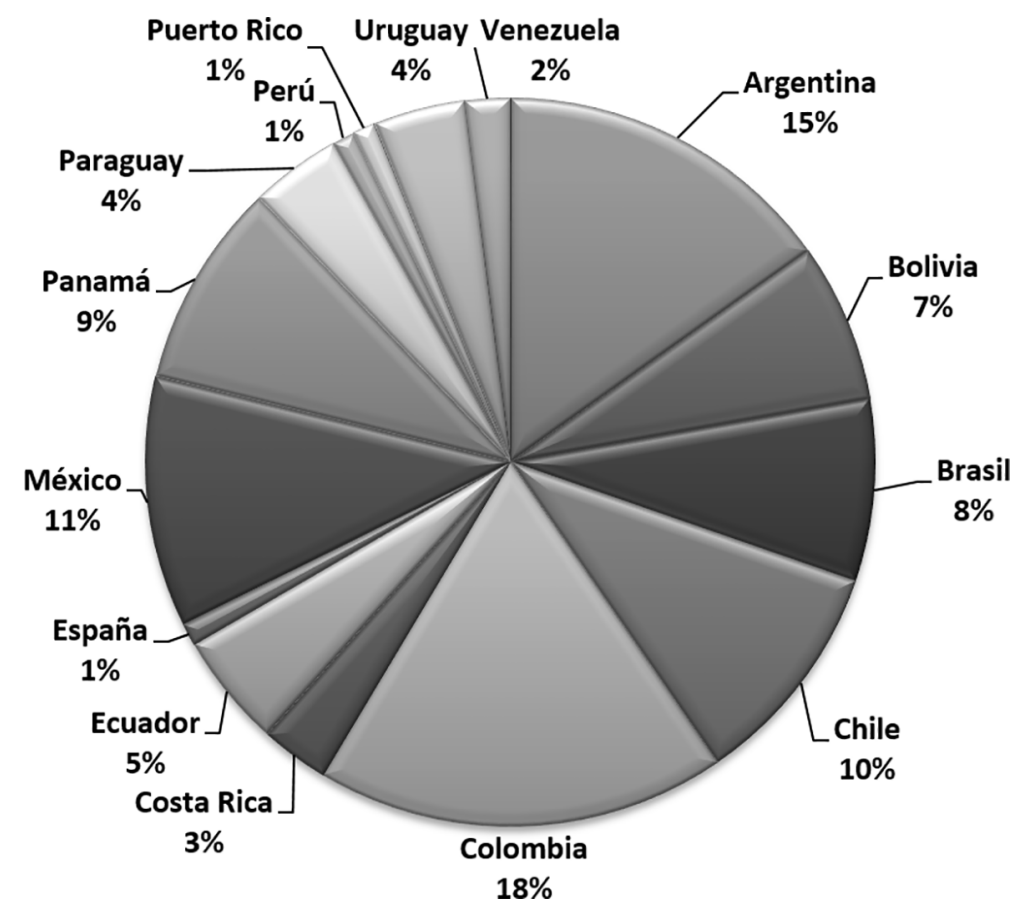

Gráfica 1. Representación (\%) de los países en las respuestas a la encuesta $(n=83)$.

En la distribución por sexo se encuentra un 58,6\% mujeres y 41,4\% hombres. Según la profesión, el $99 \%$ declaró ser médico y $1 \%$ psicólogo.

El $93 \%$ de los médicos señaló que estaban vinculados a la Medicina Familiar (MF), con un $8 \%$ de residentes, $85 \%$ especialista en MF cumpliendo residencia completa. $4 \%$ de médicos generales, $2 \%$ psiquiatra y $1 \%$ de medicina interna. De los médicos de familia, la mayoría ( $83 \%$ ) trabaja como médico de atención primaria de la salud (entendida en el contexto de esta investigación como primer nivel de 
atención), mientras que un $8 \%$ contestó que eran tutores de residentes de MF. Es necesario destacar, que la pregunta estuvo orientada a la labor principal que desempeñaban los médicos familiares, razón por la cual, no se puede inferir, si los que ejercen en atención primaria de la salud, también cumplen labores de tutoría de residentes (Gráfica 2).

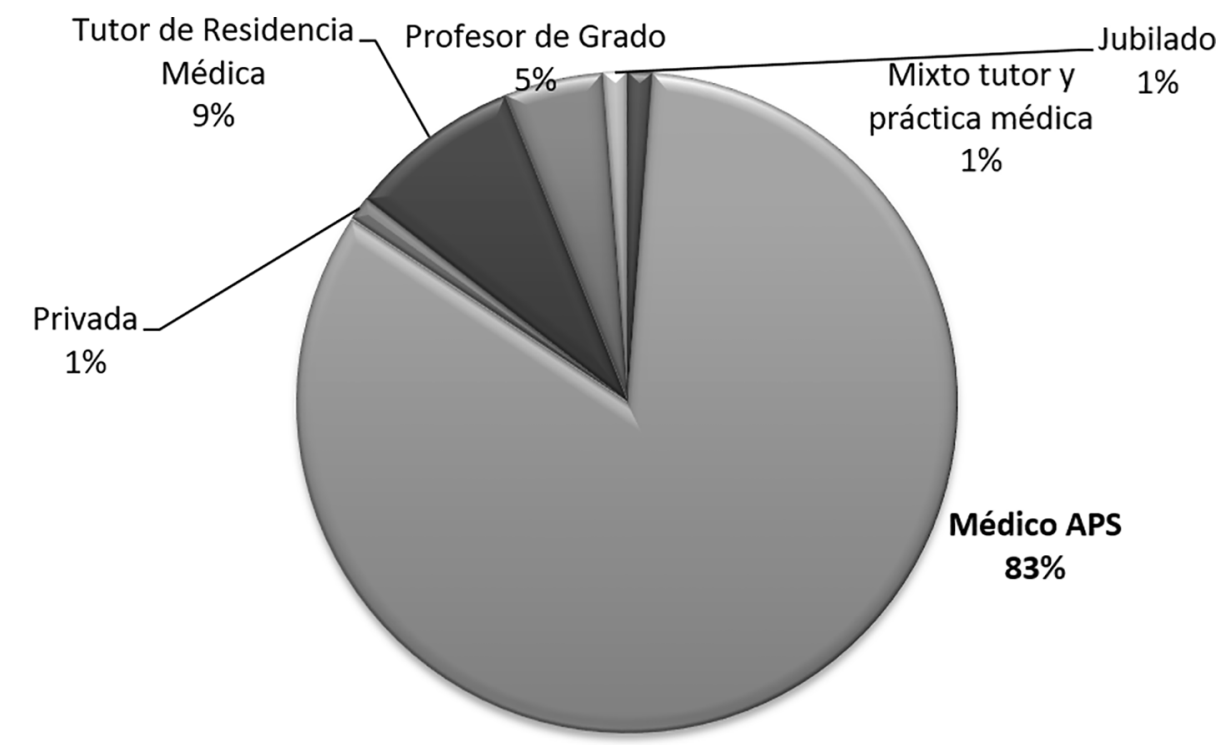

Grafica 2. Distribución (\%) de los Medicos Familiares que han contestado la encuesta segundo el labor ( $n=83$ ).

De los MF con formación de postgrado universitaria, descartando aquellos países en los que sólo respondió 1 persona, destaca que la mayoría de los MF que respondieron de Argentina (64\%), Bolivia (71\%) y Brasil (83\%), opinaron que la capacidad de los MF para abordar problemas de SM es Regular. Por su parte, los MF de México respondieron 45,5\% Regular y 45,5\% Buena, con un 9\% Mala. Los MF de países como Chile (100\%), Colombia (86\%), Costa Rica (100\%), Ecuador (80\%), Panamá (88\%), Paraguay (67\%), Uruguay $(75 \%)$ y Venezuela $(100 \%)$ declaran que la capacidad de los MF de su país es Buena y Muy buena. (Gráfica 3).

๑ Capacidad propia $\square$ Capacidad de los demás MFyC

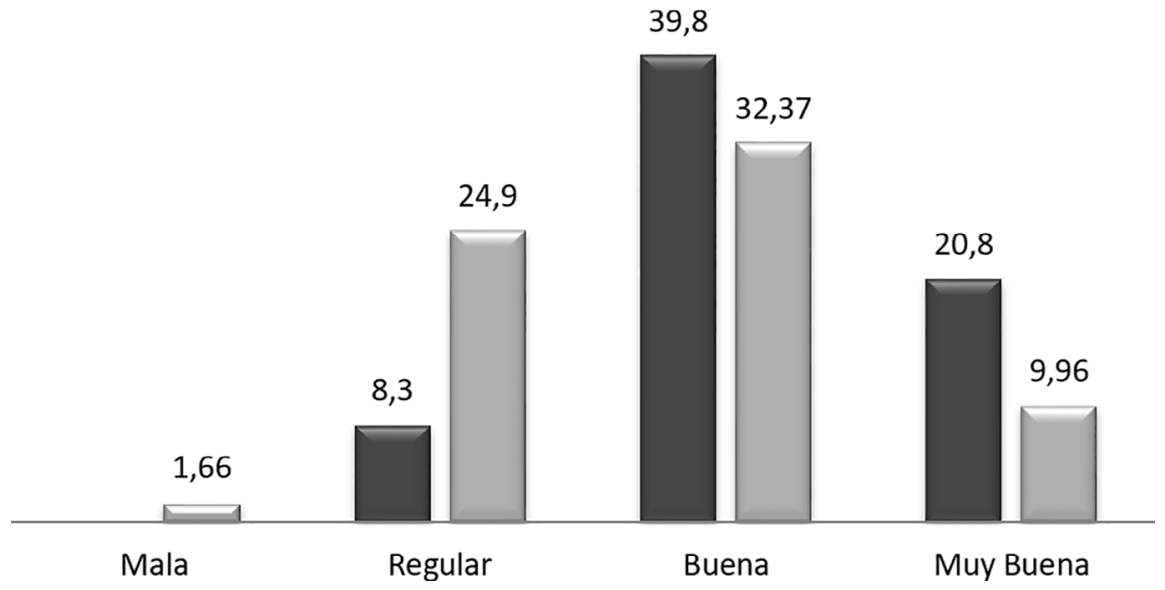

Gráfica 3. Comparación (\%) entre la percepción de los Médicos Familiares respecto su propia capacidad en manejo de Salud Mental y la capacidad de los otros MF y $(n=83)$. Fuente: Encuesta del Grupo de Trabajo 
Al hablar de la propia capacidad para abordar problemáticas de Salud Mental, se observa una mejor percepción de sí mismos. Así se dan casos como por ejemplo, los MF de Argentina perciben que un 27\% de sus colegas de MF tienen una buena y muy buena capacidad, y que ellos mismos tienen un $64 \%$ entre buena y muy buena capacidad. Así mismo, en Brasil, varía de 17\% a 100\% la autopercepción de calidad, mientras que en México fluctúa de un $45 \%$ a un $82 \%$ y Uruguay varía de un $75 \%$ a un $100 \%$. Este cambio podría hacer pensar que son más estrictos respecto de los demás médicos de familia que de sí mismos, o que hay un sesgo por mayor interés respecto de la problemática de SM al responder la encuesta y que se saben con mayores capacidades que sus colegas. Una comparación porcentual entre la percepción de los MF acerca de su propia capacidad y la de sus colegas, puede ser vista en la Grafica 3.

Por otro lado, el único psicólogo que responde la encuesta es de Argentina y declara que la capacidad y el interés de los MF en la problemática de SM es regular, lo que coincide con la percepción de los médicos de Argentina, que en su mayoría $(64,3 \%)$ consideran que es regular o mala. Sólo un $35,7 \%$ la considera como buena o muy buena. El interés sigue una tendencia similar, los encuestados declaran que el interés de los MF de su país es en su mayoría regular (78,6\%)

El número de médicos generales (MG) sin especialidad que respondió la encuesta fue muy pequeño ( 2 de Chile, 1 de Panamá y 1 de Colombia).Sin embargo, se analiza ya que pudiera orientar en una tendencia sobre la percepción que tienen de los MF. Los MG de Colombia, Panamá y uno de Chile, indicaron que la capacidad de los MF era buena en la problemática de SM, el otro de Chile, la consideró regular, y al mismo tiempo también señaló como regular su propia capacidad. Los demás calificaron como muy buena su propia capacidad sin ser MF, en contraposición con su percepción de que la capacidad de los MF es buena. Destaca que dan como justificación de ésta diferencia su propio interés en el tema de SM y, por lo tanto, su interés en capacitarse. Por otra parte, uno de ellos plantea que la capacidad de los MF se debe al tipo de atención integral que proveen.

Respecto del interés en el tema de SM, los MG lo informan como bueno y muy bueno en un $100 \%$, pero que no siempre lo pueden desarrollar por las limitaciones de tiempo de las consultas.

Los otros especialistas encuestados, 2 psiquiatras y 1 médico internista (MI), consideraron que la capacidad de los MF para detectar problemas de SM es buena. Respecto del interés de los MF en la detección y abordaje de los problemas de salud mental dicen que es bueno y muy bueno. El MI destaca que es por el conocimiento que tienen los MF de cómo los trastornos de SM inciden en la salud física. En relación a la pregunta sobre el abordaje de las personas que presentan problemas de SM en el nivel primario de atención, los tres eligen la respuesta: "Pienso que los médicos familiares son profesionales de gran relevancia porque los conceptos y herramientas de esta especialidad pueden traer nuevas miradas y posibilidades de intervención en los trastornos mentales". Sin embargo, cuando se amplía la revisión a todos los médicos, la dispersión de las respuestas y el responder a varias posibilidades a la vez, incluso contrapuestas, hace difícil observar un patrón en el resto de los médicos.

En cuanto a la frecuencia de los problemas de SM observados, de las respuestas válidas de los médicos, el 30\% indica al Trastorno de Ansiedad como el más frecuente, le sigue la depresión con 27\%, insomnio $17 \%$, alcoholismo $10 \%$, adicción a drogas ilícitas $7 \%$, trastornos alimentarios $5 \%$ y trastorno de estrés postraumático (TEPT) $4 \%$. 


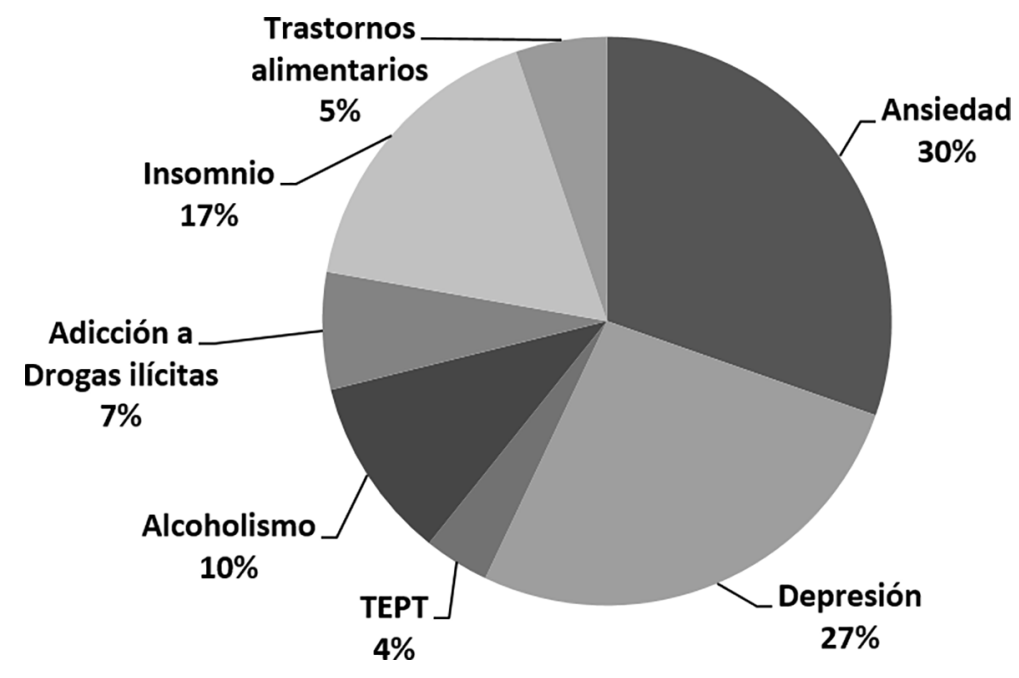

Gráfico 4. Frecuencia de los problemas de salud mental según la percepción de encuestados de lberoamérica. Marzo 2018 (n 99).

En relación a la pregunta sobre ¿qué otras patologías podría incluir como frecuentes?, en los encuestados de varios países, aparece en un 12\% la Esquizofrenia. Esto podría reflejar que los MF también están jugando un rol en el abordaje de los trastornos crónicos de SM, al igual que lo hacen por ejemplo en las enfermedades crónicas cardiovasculares. Incluso algunos lo justifican en el contexto de la cronicidad, incluyendo también como control de seguimiento al Trastorno Bipolar, al Trastorno por Déficit de Atención y otros.

Uno de los MF nos hace recordar que el Tabaquismo como cualquier adicción es un problema de SM. El no haberla incluido en el listado refleja la normalización de su uso, incluso entre nosotros los médicos.

En el mismo contexto un $5,5 \%$ nos hacen recordar que la violencia en las relaciones en general y el maltrato familiar no son infrecuentes.

En cuanto al rol del MF en los conflictos armados, un 31\% plantea que tiene competencias específicas y un especial vínculo con la población, un $21 \%$ dice que tiene competencias adecuadas para formar parte del equipo, un $17 \%$ que existe la necesidad de mayor formación y un $2 \%$ plantea que no tienen ningún rol.

En cuanto al rol del MF en los desastres naturales hay una distribución muy similar a la de los conflictos armados. Un $41 \%$ dice que el MF tiene competencias específicas y vínculo con la población, un $30 \%$ que tiene competencias adecuadas para formar parte del equipo, un $24 \%$ que es necesaria mayor capacitación, un $4 \%$ que no es su rol y $1 \%$ no lo sabe.

En cuanto a las Fortalezas que tiene el MF para actuar en dichas situaciones, se expresan: integralidad, longitudinalidad y enfoque familiar, en el contexto de la continuidad de la atención, la cercanía con la comunidad - física y afectiva - y la formación adecuada, aunque se plantea que siempre se necesita más.

En cuanto a las Barreras, hay un claro desconocimiento de la participación en políticas públicas. Un $60 \%$ no sabe o cree que los MF no participamos en definiciones de políticas públicas respecto de la Salud Mental de las personas. Un $65 \%$ no sabe o no cree que participemos en el desarrollo de estrategias de capacitación y protocolos de abordaje de problemas de Salud Mental. 


\section{Resultados del Trabajo de Grupo}

Durante el proceso de trabajo de grupo se generaron varias inquietudes respecto a la salud mental de los equipos de salud. Así mismo, surgió una marcada preocupación por la SM de los residentes de Medicina Familiar, resaltando que durante su formación, los tutores de MF deberían proporcionar las herramientas necesarias para que los futuros Médicos Familiares desarrollen las capacidades de autocuidado, y que además, les permita implementar y fortalecer la atención de SM en los centros en los que trabajen.

Por otra parte, el grupo manifestó que se debe garantizar la salud mental de los prestadores de servicios, entre ellos los MF, garantizando un espacio de descarga emocional y salarios dignos.

También se consideró prioritario que los MF participen en la creación de protocolos de intervención en equipo, que especifiquen los alcances y el límite de cada intervención. Para ello se debe fortalecer la capacitación sobre SM, para trabajar en la prevención en SM y realizar un diagnóstico precoz cuando es necesario. Estructurando además capacitaciones en intervenciones comunitarias en SM, para desarrollar la estrategia de rehabilitación basada en la comunidad y fortalecimiento de las redes de apoyo.

Se destacó además, la importancia de desarrollar competencias específicas de acuerdo al perfil epidemiológico de cada país. Orientando el quehacer del MF a generar el más alto impacto en la salud de la población.

\section{Conclusión}

Dada la alta prevalencia e incidencia de la problemática de SM y el influjo que tienen en el desarrollo de las personas, las familias y las comunidades, los MF debemos incorporar en nuestro diario quehacer, en la mirada integral, preguntas orientadas a conocer situaciones del área emocional. En cada atención, sea cual sea el motivo de ella, debemos preguntar respecto del ámbito de salud mental, no sólo “¿Cómo está usted, cómo le va?". Debemos preguntar específicamente cómo está su ánimo, ha tenido algún problema emocional en el último tiempo. Debemos recordar siempre que las personas no consultan por problemas del ámbito mental. No sólo por el estigma que aún persiste en el mundo respecto de éste tipo de problema, sino por la misma condición del problema de salud mental.

Está entonces el desafío, que es al mismo tiempo una oportunidad, de visibilizara la Medicina Familiar como una especialidad que puede y debe abordar la problemática de Salud Mental por su formación y la cercanía con la población a cargo en sus territorios.

En este contexto se realizan las siguientes recomendaciones para ser incluidas en la Carta de Cali:

En este contexto se realizan las siguientes recomendaciones para ser incluidas en la Carta de Cali:

1. Incorporar y/o fortalecer, según sea el caso, la formación en salud mental y comunitaria necesaria para los espacios de atención en los que se desenvuelven los médicos de familia, sin considerar las situaciones vitales como algo patológico, con el desarrollo de estrategias de autocuidado de las personas, sustentables tanto en el tiempo como en la capacidad financiera de los países de Iberoamérica; con el fin de desarrollar la capacidad de enfrentar situaciones estresantes de la vida diaria, que permitan desarrollar fortalezas para situaciones de emergencias y desastres.

2. Fortalecer y empoderar el trabajo comunitario con el fin de que sea la propia comunidad empoderada quien establezca redes de apoyo en problemáticas de salud mental y esté preparada 
en conjunto para enfrentar situaciones de la vida diaria y le permita desarrollar acciones inmediatas en situaciones de emergencias y desastres naturales o no.

3. Priorizar estrategias de autocuidado costo efectivas y orientadas a herramientas de desarrollo personal y de las familias, incluidos los equipos de salud, y los profesores, tutores de Medicina Familiar. De modo tal que se establezcan relaciones virtuosas orientadas a un trato cordial y constructivo. El cuerpo docente de las residencias de MF debe hacerse cargo de acciones tendientes al autocuidado de los alumnos, propendiendo a facilitar el proceso de aprendizaje y la preparación para ejercer la profesión en un medio tan complejo como son los centros de Atención Primaria de Salud o en cualquier contexto en el que se trabaje con la estrategia de APS.

\section{Referencias}

1. Resumen Ejecutivo Prevención del Suicidio OMS-OPS Nota descriptiva Enero 2018. Disponible en: http://www.who.int/mediacentre/ factsheets/fs398/es/

2. Manual para la protección y cuidado de la Salud Mental en situaciones de Emergencias y Desastres: JICA (Agencia de Cooperación Internacional del Japón) - Ministerio de Salud Chile, 2015: Disponible en: http://web.minsal.cl/wp-content/uploads/2015/09/Manualpara-la-protecci\%C3\%B3n-y-cuidado-de-la-Salud-Mental-en-situaciones-de-Emergencias-y-Desastres.pdf

3. Mario Waissbluth, EITsunami de la Patología Mental En Chile, Reflexiones de Valor Público № 1, Centro de Sistemas Públicos/Ingeniería Industrial - Universidad de Chile Octubre 2017. Disponible en: http://www.sistemaspublicos.cl/wp-content/uploads/2017/10/Reflexionesde-Valor-P\%C3\%BAblico-N\%C2\%B01.-El-Tsunami-de-la-Patolog\%C3\%ADa-Mental-en-Chile.pdf

4. Organización Mundial de la Salud (OMS). Volver a construir mejor: atención de salud mental sostenible después de una emergencia. Información general.OMS: Ginebra, 2013. Disponible en: http://apps.who.int/iris/bitstream/handle/10665/85619/WHO_MSD_MER_13.1_ spa.pdf;jsessionid=0B3B48F7C9B8CFB6037AAF7C114EFB96? sequence=1

5. McDaid, D., E. Hewlett and A. Park, "Understanding effective approaches to promoting mental health and preventing mental illness", OECD Health Working Papers, No. 97, OECD Publishing, Paris, Oct 2017. Disponible en: http://dx.doi.org/10.1787/bc364fb2-en

6. WHO-AIMS: Informe sobre los Sistemas de Salud de Salud Mental en América Latina y El Caribe, OPS 2013. Disponible en:http://www. paho.org/per/images/stories/FtPage/2013/WHO-AIMS.pdf

7. WHO-AIMS (Assessment Instrument for Mental Health Systems): Informe sobre los Sistemas de Salud de Salud Mental en América Latina y El Caribe, OPS 2013; Disponible en: http://www.paho.org/per/images/stories/FtPage/2013/WHO-AIMS.pdf

8. Organización Panamericana de la Salud. Guía de intervención humanitaria mhGAP (GIH-mhGAP). El manejo clínico de los trastornos mentales neurológicos y por uso de sustancias en las emergencias humanitarias. Washington, DC : OPS, 2016. Disponible: http://iris. paho.org/xmlui/bitstream/handle/123456789/28418/9789275319017_spa.pdf

9. Organización Mundial de la Salud, Enfrentando Problemas Plus (EP+): Ayuda psicológica individual para adultos afectados por la angustia en comunidades expuestas a adversidad. (Versión genérica de prueba de mercado 1.0). Ginebra, OMS, 2016. http://apps.who.int/iris/ bitstream/handle/10665/259696/WHO-MSD-MER-16.2-spa.pdf?sequence=1 